\title{
Artigo/Article
}

\section{Mortalidade por leishmaniose visceral: aspectos clínicos e laboratoriais}

\author{
Mortality due to visceral leishmaniasis: clinical and laboratory characteristics
}

\author{
Janaina Michelle de Oliveira ${ }^{1}$, Ana Claudia Fernandes ${ }^{2}$, Maria Elizabeth Cavalheiros Dorval ${ }^{3}$, Túlia Peixoto Alves ${ }^{4}$, \\ Thiago Dias Fernandes ${ }^{2}$, Elisa Teruya Oshiro ${ }^{4}$ e Ana Lúcia Lyrio de Oliveira ${ }^{5}$
}

\begin{abstract}
RESUMO
Introdução: A leishmaniose visceral é uma doença infecciosa sistêmica de ampla distribuição geográfica, caracterizada pelo alto potencial de letalidade. Visando contribuir com a redução da mortalidade, bem como auxiliar profissionais da saúde no manejo clínico dos pacientes portadores desse agravo, este trabalho teve como objetivo investigar as características clínicas e laboratoriais dos casos que evoluíram para o êxito letal em hospitais de Campo Grande, MS, nos anos de 2003 a 2008. Métodos: Foram analisados 55 prontuários de pacientes que tiveram a leishmaniose visceral como causa de óbito. Resultados: Dos 55 pacientes estudados, 37 eram procedentes do município de Campo Grande, sendo 41 (74,5\%) do sexo masculino, com predominância da faixa etária acima dos 40 anos. Quanto ao quadro clínico, a febre esteve presente em $89,1 \%$ dos casos. A duração da doença desde o início dos sintomas até a hospitalização variou em média 78,2 dias. A leucopenia ocorreu em $85,5 \%$ dos pacientes. Comorbidades estiveram presentes em $39(70,9 \%)$ pacientes, sendo a desnutrição e o etilismo as mais frequentes. A confirmação do diagnóstico ocorreu em média 6,7 dias após a internação. $\mathrm{O}$ antimoniato pentavalente foi a droga mais utilizada, com $87,5 \%$ dos pacientes apresentando algum tipo de reação adversa. Infecções bacterianas ocorreram em 36 pacientes e, em 27 (49\%), foram uma das causas do óbito. Conclusões: Os dados indicam que a identificação precoce dessas características clínicas e laboratoriais no primeiro atendimento ao paciente é de fundamental importância para se reduzir a mortalidade por meio da instituição de medidas terapêuticas e profiláticas eficazes.
\end{abstract}

Palavras-chaves: Leishmaniose visceral. Mortalidade. Mato Grosso do Sul.

\begin{abstract}
Introduction: Visceral leishmaniasis is a systemic infectious disease of broad geographical distribution, characterized by high potential for lethality. With the purpose of contributing towards reducing mortality and helping healthcare professionals in clinical management of patients with this disease, this paper aimed to investigate the clinical and laboratory characteristics of cases with a fatal outcome in hospitals in Campo Grande, Mato Grosso do Sul, between 2003 and 2008. Methods: Fifty-five medical files on patients who died due to visceral leishmaniasis were analyzed. Results: Among the 55 patients studied, 37 were from the municipality of Campo Grande; $41(74.5 \%)$ were males; and age over 40 years predominated. The patients presented with fever in $89.1 \%$ of the cases. The duration of the illness from the onset of symptoms to hospitalization was 78.2 days on average. Leukopenia was seen in $85.5 \%$ of the patients. Comorbidities were present in 39 (70.9\%) patients; malnutrition and alcoholism were the most frequent of these. Confirmation of the diagnosis occurred on average 6.7 days after admission. Pentavalent antimoniate was the drug most used, and $87.5 \%$ of the patients presented some type of adverse reaction. Bacterial infections occurred in 36 patients and were one of the causes of death in 27 (49\%). Conclusions: The data showed that early identification of these clinical and laboratory characteristics, at the time when patients are first attended, is extremely important for reducing mortality through instituting efficient therapeutic and prophylactic measures.
\end{abstract}

Key-words: Visceral leishmaniasis. Mortality. Mato Grosso do Sul.

1. Programa de Pós-Graduação em Doenças Infecciosas e Parasitárias, Faculdade de Medicina, Universidade Federal de Mato Grosso do Sul, Campo Grande, MS. 2. Departamento de Medicina, Faculdade de Medicina, Universidade Federal de Mato Grosso do Sul, Campo Grande, MS. 3. Departamento de Patologia, Universidade Federal de Mato Grosso do Sul, Campo Grande, MS. 4. Departamento de Clínica Médica, Universidade Federal de Mato Grosso do Sul, Campo Grande, MS. 5. Departamento de Pediatria, Universidade Federal de Mato Grosso do Sul, Campo Grande, MS.

Endereço para correspondência: MsC. Janaina Michelle de Oliveira. Rua Golf 2043, Taveirópolis, 79090360 Campo Grande, MS.

Tel: 5567 3363-2815; Fax: 5567 3363-2815

e-mail: janainamoliveira@gmail.com

Recebido para publicação em 02/10/2009

Aceito em 29/01/2010

\section{INTRODUÇÃO}

A leishmaniose visceral (LV) é uma zoonose caracterizada pelo envolvimento sistêmico que afeta milhões de pessoas em regiões tropicais e subtropicais do globo ${ }^{1,2}$. Há uma incidência estimada de 500.000 casos novos e 50.000 mortes a cada ano no mundo, com números claramente em ascensão ${ }^{3,4}$.

É uma doença espectral, cuja apresentação clínica varia de formas assintomáticas até o quadro clássico da parasitose, evidenciado pela presença de febre, anemia, hepatoesplenomegalia, além de tosse seca, leucopenia e hipergamaglobulinemia. Outras manifestações clínicas se desenvolvem com a progressão da doença, em especial a diarréia, icterícia, vômito e o edema periférico que dificultam o diagnóstico diferencial com outras patologias, retardando sua identificação ${ }^{5,6}$.

A associação de comorbidades como a desnutrição, o diagnóstico tardio da doença e a presença de complicações, como as infecções bacterianas principalmente por Staphylococcus aureus e Pseudomonas aeruginosa e as hemorragias concorrem para o aumento da letalidade por este agravo ${ }^{7,8}$.

Em Mato Grosso do Sul, tem-se verificado um incremento no número de casos da LV, com ampliação de sua área de ocorrência. Até 1995, a doença estava restrita ao município de Corumbá, porém, se expandiu para as localidades adjacentes, apresentando-se, no momento, em 56 dos 78 municípios do estado ${ }^{9,10}$.

Em Campo Grande, capital do estado, onde a doença teve seu início no ano de 2001, até o momento foram registrados 984 casos autóctones, com 69 óbitos, resultando em coeficiente de letalidade de $7 \%$ e de mortalidade de 9,1 por $100 \mathrm{mil}$ habitantes, demonstrando a gravidade do problema e a endemicidade da doença na cidade ${ }^{9}$.

Uma vez que são escassos os relatos envolvendo os fatores associados à evolução clínica desfavorável dos portadores de LV, o presente estudo teve por objetivo identificar as características clínicas e laboratoriais de pacientes que foram a óbito em quatro hospitais de Campo Grande, Mato Grosso do Sul no período de 2003 a 2008. 


\section{MÉTODOS}

Realizou-se um estudo retrospectivo, no período entre janeiro de 2003 e dezembro de 2008, em prontuários de pacientes que evoluíram para o óbito por LV (CID B55) nos seguintes hospitais de Campo Grande, MS: Hospital Universitário Maria Aparecida Pedrossian (NHU-UFMS), Hospital Regional Rosa Pedrossian, Hospital São Julião e Santa Casa de Misericórdia.

Após a confirmação dos pacientes no Serviço de Vigilância Epidemiológica (SVE) do município, realizou-se uma revisão sistemática dos prontuários com preenchimento de um formulário contendo dados de identificação, procedência, queixas principais, tempo de duração da doença, diagnóstico realizado, presença de outras patologias, exames laboratoriais e tratamento.

Foram incluídos no estudo todos os pacientes que foram a óbito nos referidos anos e que apresentavam diagnóstico confirmado pela pesquisa do parasito em aspirado de medula óssea, mielocultura ou reação de imunofluorescência indireta (RIFI) para anticorpos antiLeishmania (considerados títulos positivos $\geq 1: 80$ ). Foram excluídos pacientes que não tiveram os prontuários liberados pela direção dos hospitais, aqueles com os prontuários extraviados, os que não apresentavam informações suficientes para a realização da pesquisa e os que não constavam como internados nos referidos hospitais.

Visto que os dados são secundários, foram considerados desnutridos os pacientes em cujos prontuários constavam o termo desnutrido e/ou índice de massa corporal (IMC). Para classe pediátrica, além do termo, considerou-se também o critério de Gomez.

Para a definição de alterações laboratoriais, consideraram-se os seguintes parâmetros normais: hemoglobina $(\mathrm{Hb})$ (crianças: 11 a $13 \mathrm{~g} / \mathrm{dL}$; adultos: 12 a $16 \mathrm{~g} / \mathrm{dL}$ ), hematócrito (crianças: 35 a 38\%; adultos: 35 a $55 \%)$, leucócitos ( 5.000 a $\left.10.000 / \mathrm{mm}^{3}\right)$, bastonetes (0 a 4\%), segmentados ( 50 a $65 \%$ ), linfócitos (20 a 30\%), plaquetas $\left(150.000\right.$ a $450.000 / \mathrm{mm}^{3}$ ), proteínas totais (crianças: 5,0 a $7,5 \mathrm{~g}$ / $\mathrm{dL}$; adultos: 6,0 a $8,0 \mathrm{~g} / \mathrm{dL}$ ), creatinina (crianças: até $1,0 \mathrm{mg} / \mathrm{dL}$; adultos: 0,6 a 1,3mg/dL), alanina aminotransferase (ALT) (crianças: até $50 \mathrm{UI} / \mathrm{L}$; adultos: 30 a $65 \mathrm{UI} / \mathrm{L})$, aspartato aminotransferase (AST) (crianças: até 80UI/L; adultos: 15 a 37UI/L), amilase (25 a 115UI/L) e lipase (10 a 140UI/L) ${ }^{11}$.

Os dados foram organizados e analisados descritivamente com o auxílio dos programas Microsoft Office Excel 2003 e Epi Info ${ }^{\text {Ts }}$ version 3.5.1 respectivamente. A fim de verificar possíveis associações entre as variáveis do estudo foram utilizados os testes de Wilcoxon e o teste $t$ Student pareado, ao nível de significância de $5 \%(p<0,05)$ por meio dos softwares BioEstat 4.0 e SPSS (SPSS for Windows version 13.0).

\section{Ética}

Este trabalho foi aprovado pelo Comitê de Ética em Pesquisa da Universidade Federal de Mato Grosso do Sul (UFMS).

\section{RESULTADOS}

Foram estudados 55 pacientes que representaram $76,4 \%$ do total de pacientes que morreram por LV entre janeiro de 2003 e dezembro de 2008. Destes, 41 (74,5\%) eram do sexo masculino e 14 (25,5\%) do feminino, sendo esta diferença estatisticamente significativa de acordo com o teste de proporção binomial ( $\mathrm{M}: \mathrm{F}=1: 1)(p<0,0001)$. A média etária foi de 46,7 anos, com variação de seis meses a 93 anos (Tabela 1).
A procedência dos pacientes foi predominantemente da Cidade de Campo Grande ( 37 casos), sendo os demais provenientes dos municípios de Aquidauana (3), Três Lagoas (3), Terenos (2), Água Clara, Amambaí, Bonito, Chapadão do Sul, Corumbá, Fátima do Sul, Miranda, Ponta Porã, Ribas do Rio Pardo e Sete Quedas (um caso cada).

A duração da doença desde o início dos sintomas até a hospitalização variou de 1 a 365 dias, com média de 78,2 dias e mediana de 50 dias e em $60 \%$ dos casos os sintomas evoluíram em período inferior a 60 dias.

Na Tabela 2, entre as principais manifestações clínicas, destacaramse a febre em $89,1 \%$ dos casos, seguida por emagrecimento $(74,5 \%)$, esplenomegalia (71\%), hepatomegalia (71\%) e palidez (69\%).

Os dados hematológicos referentes às séries vermelha, branca e plaquetária, no momento da admissão hospitalar e no óbito dos pacientes, estão apresentados na Tabela 3. A mediana da $\mathrm{Hb}$

TABELA 1 - Distribuição por faixa etária e sexo dos pacientes que evoluíram para o óbito por leishmaniose visceral em hospitais do município de Campo Grande - MS, de janeiro de 2003 a dezembro de 2008.

\begin{tabular}{|c|c|c|c|c|c|c|c|c|}
\hline \multirow{2}{*}{$\begin{array}{l}\text { Idade } \\
\text { (anos) }\end{array}$} & \multicolumn{2}{|c|}{ Fem } & \multicolumn{2}{|c|}{ Masc } & \multicolumn{2}{|c|}{ Total } & \multirow{2}{*}{$\begin{array}{r}\text { Porcentagem } \\
\text { acumulativa }\end{array}$} & \multirow[b]{2}{*}{ Letalidade } \\
\hline & $\mathrm{n}^{\mathrm{o}}$ & $\%$ & $\mathrm{n}^{\circ}$ & $\%$ & $\overline{\mathrm{n}^{\circ}}$ & $\%$ & & \\
\hline$<1$ & - & - & 2 & 4,9 & 2 & 3,6 & 3,6 & $3,1 / 100$ \\
\hline $1 \mathrm{H} 4$ & 4 & 28,6 & 2 & 4,9 & 6 & 10,9 & 14,5 & $3,5 / 100$ \\
\hline $5 \mathrm{H} 9$ & - & - & - & - & - & - & - & - \\
\hline $10 \mathrm{H} 19$ & - & - & 1 & 2,4 & 1 & 1,8 & 16,3 & $1,4 / 100$ \\
\hline $20 \mathrm{H} 39$ & 3 & 21,4 & 5 & 12,2 & 8 & 14,6 & 30,9 & $4,1 / 100$ \\
\hline $40 \mathrm{H} 59$ & 2 & 14,3 & 20 & 48,8 & 22 & 40 & 70,9 & $14,4 / 100$ \\
\hline$>60$ & 5 & 35,7 & 11 & 26,8 & 16 & 29,1 & 100,0 & $20,0 / 100$ \\
\hline Total & 14 & 100,0 & 41 & 100,0 & 55 & 100,0 & 100,0 & $6,6 / 100$ \\
\hline
\end{tabular}

Fem: feminino, Masc: masculino.

TABELA 2 - Frequência das manifestações clínicas observadas na admissão hospitalar em pacientes que evoluíram para o óbito por leishmaniose visceral em hospitais do município de Campo Grande - MS, de janeiro de 2003 a dezembro de 2008 .

\begin{tabular}{|c|c|c|c|}
\hline \multirow[b]{2}{*}{ Manifestações clínicas } & \multicolumn{3}{|c|}{ Pacientes } \\
\hline & $\mathrm{n}^{\mathrm{o}}$ & $\%$ & $\mathrm{IC}_{95 \%}$ \\
\hline Febre & 49 & 89,1 & $80,9-97,3$ \\
\hline Emagrecimento & 41 & 74,5 & $63,0-86,1$ \\
\hline Esplenomegalia & 39 & 71,0 & $58,9-82,9$ \\
\hline Hepatomegalia & 39 & 71,0 & $58,9-82,9$ \\
\hline Palidez & 38 & 69,0 & $56,9-81,3$ \\
\hline Hiporexia & 36 & 65,5 & $52,9-78,0$ \\
\hline Astenia & 32 & 58,1 & $45,1-71,2$ \\
\hline Tosse & 27 & 49,0 & $35,9-62,3$ \\
\hline Dor abdominal & 23 & 41,8 & $28,8-54,9$ \\
\hline Edema & 22 & 40,0 & $27,1-52,9$ \\
\hline Fenômenos hemorrágicos & 18 & 32,7 & $20,3-45,1$ \\
\hline Adinamia & 16 & 29,0 & $17,1-41,1$ \\
\hline Náusea/vômito & 16 & 29,0 & $17,1-41,1$ \\
\hline Diarréia & 16 & 29,0 & $17,1-41,1$ \\
\hline Mialgia & 13 & 23,6 & $12,4-34,9$ \\
\hline Icterícia & 11 & 20,0 & $9,4-30,6$ \\
\hline Sudorese & 11 & 20,0 & $9,4-30,6$ \\
\hline Calafrios & 3 & 5,5 & - \\
\hline Alterações neurológicas & 2 & 3,6 & - \\
\hline
\end{tabular}

$\mathrm{IC}_{95 \%}$ : intervalo de confiança. 
na internação foi de $7,5 \mathrm{~g} / \mathrm{dL}$ e no óbito $7,7 \mathrm{~g} / \mathrm{dL}$, sendo que em dez pacientes os níveis estavam abaixo de $5,5 \mathrm{~g} / \mathrm{dL}$. A contagem máxima de leucócitos na admissão foi de $10.600 / \mathrm{mm}^{3}$; porém, $47(85,5 \%)$ pacientes deram entrada apresentando valores abaixo de $5.000 / \mathrm{mm}^{3}$. No óbito, a máxima de leucócitos foi de $26.200 /$ $\mathrm{mm}^{3}$, apresentando aumento significativo $(\mathrm{p}=0,007)$. A mediana de bastonetes, segmentados e linfócitos na admissão foi de 7\%, 50\%, e $32 \%$ respectivamente, e quando analisados no óbito, os valores foram de $9 \%, 58 \%$ e $36 \%$.

Na Tabela 4, encontram-se os valores bioquímicos estudados. À admissão, as dosagens séricas de albumina foram realizadas em 33 pacientes e, em apenas $4(12,1 \%)$, os níveis séricos estavam dentro dos valores de referência $(3,5$ a 5,5g/dL). A elevação de AST ocorreu em 31 (79,5\%) pacientes dos 39 que realizaram o exame na admissão. Já para ALT, os níveis séricos estavam normais em 25 (65,6\%) dos 37 submetidos ao exame. Os níveis de creatinina e amilase no óbito elevaram-se significativamente $(\mathrm{p} \leq 0,05)$.

Dos 55 pacientes, 39 (70,9\%) apresentaram na admissão uma ou mais patologias associadas, conforme pode ser observado na Tabela 5. Menciona-se o diagnóstico de outras morbidades tais como, asma, bloqueio do ramo direito do $2^{\circ}$ grau (BRD II grau), citomegalovírus (CMV), doença de Addison, doença pulmonar obstrutiva crônica (DPOC), hemoglobinopatia, leucemia mielóide aguda (LMA), mieloma múltiplo e vírus T-linfotrópico humano (HTLV), todas com apenas uma ocorrência.

Em relação ao diagnóstico, a pesquisa direta do parasito em aspirado de medula óssea foi realizada em 49 pacientes, sendo positiva em $45(91,8 \%)$, um paciente apresentou apenas a mielocultura positiva e em nove casos o diagnóstico só foi possível por sorologia (RIFI). O tempo decorrido entre a admissão e o diagnóstico variou de 1 a 33 dias, com média de 6,7 dias e mediana de 4 dias.

Foram tratados $47(85,5 \%)$ pacientes; os demais $(8 / 14,5 \%)$ morreram antes da terapêutica ter sido instituída, tendo como prováveis causas, a presença de comorbidades, demora na assistência médica e/ou diagnóstico tardio, que nestes pacientes ocorreu 3,8 dias em média após a internação. Dos pacientes tratados, 24 receberam antimoniato de meglumina e 22 desoxicolato de anfotericina $B$, mencionando-se que em sete desses pacientes a opção por esta droga ocorreu após resposta insatisfatória ao antimoniato, caracterizada pelo não desaparecimento dos sinais e sintomas e/ou piora do quadro clínico. A anfotericina B lipossomal foi o tratamento de escolha em oito pacientes pelos sinais de gravidade apresentados como insuficiência renal crônica (IRC), e ainda etilismo e cirrose hepática associados com comprometimento renal. $\mathrm{O}$ tempo médio de internação foi de 14,9 dias.

Uma ou mais reações adversas ocorreram em 32 (58,2\%) pacientes ao longo do tratamento. Desses, 21 apresentaram reações ao antimoniato de meglumina, sendo as mais frequentes: insuficiência renal aguda (IRA) $(53,4 \%)$, insuficiência hepática $(33,3 \%)$, alterações cardíacas $(28,6 \%)$, pancreatite $(28,6 \%)$ e alterações da consciência (19\%). Outras reações foram náusea e vômito $(5 / 21)$, alergia (exantema) (3/21), hepatite medicamentosa $(3 / 21)$ e um paciente apresentou prurido generalizado no momento da infusão da droga. Reações à anfotericina $\mathrm{B}$ ocorreram em seis pacientes, sendo as mais relatadas a insuficiência hepática (4/6), IRA (3/6), alterações cardíacas (2/6), alterações da consciência (2/6) e flebite (1/6). Anfotericina B lipossomal ocasionou reações em 5 pacientes, com IRA $(3 / 5)$, alterações cardíacas $(2 / 5)$ e flebite $(1 / 5)$ as mais descritas.

Infecções foram diagnosticadas, antes ou durante o período de internação, em $36(65,5 \%)$ pacientes. Destaca-se a ocorrência de sepse em 24 (66,7\%), pneumonia em 23 (63,9\%) e desses, 12 cursaram com sepse. Infecções do trato urinário ocorreram em dois $(5,5 \%)$ e otite média aguda em um paciente.

Dentre as causas que contribuíram para o óbito, as infecções bacterianas foram as principais, ocorrendo em 27 (49\%) pacientes. Insuficiência respiratória foi constatada em 17 (30,9\%), IRA em 11 (20\%), insuficiência hepática em oito (14,5\%), hemorragia (digestiva, pulmonar, epistaxe e gengivorragia) em seis (10,9\%) e arritmia cardíaca em dois casos (3,6\%). Infecções fatais incluíram pneumonia e choque séptico.

TABELA 3 - Resultados dos exames hematológicos dos pacientes que evoluíram para o óbito por leishmaniose visceral em hospitais do município de Campo Grande - MS, de janeiro de 2003 a dezembro de 2008.

\begin{tabular}{|c|c|c|c|c|c|c|c|}
\hline \multirow[b]{2}{*}{ Exames } & \multicolumn{2}{|c|}{ Mediana \pm DP } & \multicolumn{2}{|c|}{ Mínimo } & \multicolumn{2}{|c|}{ Máxima } & \multirow[b]{2}{*}{$p$} \\
\hline & $\mathrm{adm}$ & óbito & $\mathrm{adm}$ & óbito & $\mathrm{adm}$ & óbito & \\
\hline $\mathrm{Hb}(\mathrm{g} / \mathrm{dL})^{(1)}$ & $7,5 \pm 2,4$ & $7,7 \pm 1,6$ & 2,7 & 1,9 & 12,8 & 13,9 & 0,4580 \\
\hline $\mathrm{Ht}(\%)^{(1)}$ & $23 \pm 7,2$ & $23 \pm 4,7$ & 8,7 & 6,2 & 38 & 40 & 0,7180 \\
\hline Leuc $\left(\mathrm{mm}^{3}\right)^{(2)}$ & $2.200 \pm 1751,4$ & $2.700 \pm 6050,8$ & 272 & 350 & 10.600 & 26.200 & 0,0070 \\
\hline $\operatorname{Plaq}\left(\mathrm{mm}^{3}\right)^{(2)}$ & $50.000 \pm 70562$ & $39.000 \pm 66410$ & 2.000 & 2.000 & 357.000 & 284.000 & 0,3756 \\
\hline
\end{tabular}

DP: desvio padrão, adm: admissão, Hb: hemoglobina, Ht: hematócrito, Leuc: leucócitos, Plaq: plaquetas, ${ }^{(1)}$ Teste $t$ Student pareado, ${ }^{(2)}$ Teste Wilcoxon.

TABELA 4 - Resultados dos exames bioquímicos dos pacientes que evoluíram para o óbito por leishmaniose visceral em hospitais do município de Campo Grande - MS, de janeiro de 2003 a dezembro de 2008.

\begin{tabular}{|c|c|c|c|c|c|c|c|}
\hline \multirow[b]{2}{*}{ Exames } & \multicolumn{2}{|c|}{ Mediana \pm DP } & \multicolumn{2}{|c|}{ Mínimo } & \multicolumn{2}{|c|}{ Máxima } & \multirow[b]{2}{*}{$p$} \\
\hline & $\mathrm{adm}$ & óbito & adm & óbito & $\mathrm{adm}$ & óbito & \\
\hline Prot totais $(\mathrm{g} / \mathrm{dL})^{(1)}$ & $6,8 \pm 1,9$ & $6,9 \pm 2,0$ & 4,4 & 4,1 & 12,2 & 11 & 0,0600 \\
\hline Creat $(\mathrm{mg} / \mathrm{dL})^{(2)}$ & $1,1 \pm 1,4$ & $1,8 \pm 1,9$ & 0,2 & 0,2 & 5,0 & 7,4 & 0,0002 \\
\hline $\operatorname{ALT}(\mathrm{UI} / \mathrm{L})^{(2)}$ & $42 \pm 92,2$ & $55 \pm 313,9$ & 10 & 23 & 451 & 2.120 & 0,1752 \\
\hline $\operatorname{AST}(\mathrm{UI} / \mathrm{L})^{(2)}$ & $80 \pm 125,9$ & $94 \pm 991,8$ & 19 & 26 & 577 & 4.814 & 0,4470 \\
\hline Amilase (UI/L) $)^{(2)}$ & $40 \pm 58,6$ & $166 \pm 94,9$ & 14 & 10 & 626 & 3516 & 0,0160 \\
\hline Lipase $(\mathrm{UI} / \mathrm{L})^{(1)}$ & $199,5 \pm 64,6$ & $863 \pm 339,8$ & 116 & 140 & 327 & 22.389 & 0,1100 \\
\hline
\end{tabular}

Prot totais: proteínas totais, adm: admissão, DP: desvio padrão, Creat: creatinina, ALT: alanina aminotransferase, AST: aspartato aminotransferase, ${ }^{(1)}$ teste $t$ Student pareado, ${ }^{(2)}$ Teste Wilcoxon. 
TABELA 5 - Frequência das comorbidades relatadas na admissão hospitalar dos pacientes que evoluíram para o óbito por leishmaniose visceral em hospitais do município de Campo Grande - MS, de janeiro de 2003 a dezembro de 2008.

\begin{tabular}{lrrr}
\hline & \multicolumn{3}{c}{ Pacientes } \\
\cline { 2 - 4 } Comorbidades & $\mathrm{n}^{\circ}$ & $\%$ & $\mathrm{IC}_{95 \%}$ \\
\hline Não apresentavam patologias & 16 & 29,1 & $17,1-41,1$ \\
Desnutrição & 18 & 32,7 & $20,3-45,1$ \\
Etilismo & 12 & 21,8 & $10,9-32,7$ \\
Diabetes mellitus & 9 & 16,4 & $6,6-26,1$ \\
HAS & 9 & 16,4 & $6,6-26,1$ \\
IRC & 7 & 12,7 & $3,9-21,5$ \\
HIV/SIDA & 5 & 9,1 & - \\
Cirrose hepática & 3 & 5,5 & - \\
Tuberculose & 3 & 5,5 & - \\
Hepatite C & 2 & 3,6 & - \\
ICC & 2 & 3,6 & - \\
Outras & 9 & 16,4 & $6,6-26,1$ \\
\hline
\end{tabular}

HAS: hipertensão arterial sistêmica, IRC: insuficiência renal crônica, HIV/SIDA vírus da imunodeficiência humana/síndrome da imunodeficiência adquirida, ICC: insuficiência cardíaca congestiva.

\section{DISCUSSÃO}

O processo de expansão e urbanização da LV vislumbrado por Deane, em $1956^{12}$, hoje é fato consolidado em muitas localidades brasileiras, incluindo a área geográfica da presente casuística ${ }^{10,13,14}$.

Os pacientes na sua totalidade eram procedentes de diferentes municípios do Estado de Mato Grosso do Sul, o que demonstra o processo de expansão geográfica da doença, sua instalação e endemicidade na região que no período de 2003 a 2008 registrou 1.517 casos $^{9}$.

A Cidade de Campo Grande que no período estudado apresentou 828 casos da parasitose foi responsável pelo maior número de casos na presente casuística. A abertura de avenidas acompanhando os cursos de córregos e a derrubada da vegetação para a construção de casas populares, dentre outros fatores, podem ser citados como contribuintes para o aumento da densidade vetorial, assim como a instalação e manutenção da doença no município ${ }^{10,15}$.

Entre os pacientes estudados, observou-se predomínio da doença no sexo masculino $(74,5 \%)$, o que está de acordo com outros autores ${ }^{10,16}$ que justificam o fato pela presença destes em áreas que oferecem maior risco, por moradia, trabalho ou lazer, porém, sabe-se que a infecção pode ocorrer de forma igual, sem preferência por sexo ${ }^{17}$. A possível associação dessa susceptibilidade masculina a fatores genéticos já foi comentada em um estudo realizado no Piauí, embora as causas ainda não tenham sido devidamente esclarecidas ${ }^{18}$. Na presente casuística, não foi possível estabelecer qualquer relação entre o sexo masculino e a doença, por falta de informações passíveis de análise.

A letalidade por LV prevaleceu em indivíduos acima de 40 anos (69\%), reafirmando a observação que adultos com idade igual ou superior a 45 anos apresentam maior risco de morrer possivelmente em função do declínio imunológico nessa faixa etária ${ }^{16,19}$.

A grande variabilidade em relação ao tempo de evolução da doença encontrada é compatível com dados da literatura que descrevem períodos que variam de dois a seis meses em média ${ }^{5,20}$. Duração prolongada dos sintomas e consequentemente, a demora no diagnóstico e tratamento dos pacientes com LV tem sido identificada como fator de risco para morte ${ }^{21}$.
Neste estudo, o diagnóstico laboratorial ocorreu em média seis dias após a internação e considerando a elevada positividade do exame parasitológico, é possível concluir que o mesmo não foi fator contribuinte para o êxito letal na doença, reiterando a necessidade de capacitação dos profissionais para a suspeita clínica da doença e adequada conduta do paciente.

As manifestações clínicas apresentadas pelos pacientes estão em consonância com as encontradas na literatura ${ }^{5,6,22}$. Febre, aumento do fígado e do baço, palidez, hiporexia, tosse e dor abdominal são sintomas clássicos da doença que estiveram presentes em quase todos os casos no momento da admissão tendo em vista que é durante este estágio que a maioria procura por assistência médica.

Os pacientes apresentaram ainda, emagrecimento, vômitos e diarréia, sintomas estes também relatados em outros estudos e, quando associados, podem constituir marcadores de mau prognóstico ${ }^{5,10,23}$.

Fenômenos hemorrágicos ocorreram em $32,7 \%$ dos pacientes, e na análise dos dados hematológicos a mediana de plaquetas, no momento da admissão, foi de $50.000 / \mathrm{mm}^{3}$ corroborando o achado de Alves, em 1996, citado por Queiroz, Alves e Correia ${ }^{20}$, de que a plaquetopenia é um achado frequente na $\mathrm{LV}$, presente em 50 a $70 \%$ dos casos e pode ser um fator preditor de hemorragia grave nos pacientes em que a contagem de plaquetas é inferior a $150.000 / \mathrm{mm}^{3}$.

A diminuição dos níveis de hemoglobina detectada em $89 \%$ dos casos pode ter origem multifatorial, podendo decorrer de bloqueio de produção da medula, sequestro esplênico, hemólise imune, hemorragia, doenças parasitárias e carência de ferro ${ }^{5,20,23}$.

A leucopenia é relatada com grande frequência em estudos clínicos e laboratoriais envolvendo pacientes com LV. Esteve presente em $85,5 \%$ dos casos no momento da internação, e assim como em outros portadores da doença, é possível que a esplenomegalia registrada em $71 \%$ dos pacientes tenha contribuído para esta alteração $0^{21,24,25}$. Cita-se ainda a hipoplasia ou depressão medular, que usualmente está presente na LV seja também responsável por esta redução de leucócitos ${ }^{20}$.

Neste estudo, assim como já destacado por outros autores ${ }^{26,27}$ as infecções provavelmente ocorreram em função da leucopenia e imunossupressão decorrente da própria doença ou, em alguns casos, pela presença de comorbidades.

Deve-se mencionar também, que foram diagnosticadas infecções antes da internação, uma vez que os pacientes já apresentavam ao hemograma realizado na admissão, aumento da linhagem granulocítica sugerindo a presença de um processo infeccioso. Assim, embora em alguns pacientes o êxito letal tenha ocorrido antes do início do tratamento, o que pode ser justificado pela gravidade do quadro clínico, chama a atenção o fato de que houve aumento significativo na contagem de leucócitos no óbito daqueles pacientes que receberam medicação, permitindo supor uma possível recuperação do estado de imunossupressão durante a instituição da terapia medicamentosa e/ou também a tentativa de combater o processo infeccioso pela hiperplasia medular.

Valores elevados das enzimas hepáticas no momento da admissão associado ao fato de que os pacientes não haviam recebido nenhum tipo de medicação e ainda apresentavam quadros ictéricos, sugerem o envolvimento hepático pela ação da Leishmania causando hepatite, como observado por Jerônimo e cols ${ }^{28}$.

Além da hepatite aguda, as manifestações hepáticas podem cursar com insuficiência hepática, que nesta casuística foi uma das causas para o óbito, por possível ação do parasito ou em decorrência da toxicidade das drogas utilizadas para o tratamento da $\mathrm{LV}^{29}$. 
No presente estudo, as alterações neurológicas e da consciência podem ser decorrentes da toxicidade do medicamento empregado. Em alguns casos, quadros neurológicos na LV podem ocorrer devido à insuficiência hepática e toxicidade da droga, e geralmente são progressivos passando por estágios desde sonolência até o coma ${ }^{29}$. Além disso, já foi mencionada a presença de formas amastigotas em células das meninges e do plexo coróide, assim como em encéfalo de animais e humanos e embora exista a descrição de sinais e sintomas neurológicos em seres humanos e cães portadores da parasitose, não há consenso na literatura quanto à patogênese da doença no sistema nervoso central ${ }^{10-32}$.

O aumento significativo dos níveis de amilase e o aparecimento de casos de pancreatite no decorrer do tratamento dos pacientes ratificam as afirmações de que valores anormais dos níveis séricos dessa enzima, associado ao aumento da lipase, são constantemente detectados nos portadores de LV após o início da terapia antimonial, e são causas de pancreatite, contribuindo para o óbito ${ }^{33,34}$.

A insuficiência renal aguda ocorre com frequência em pacientes com LV e está relacionada à morbidade e mortalidade na doença. No presente estudo, o seu encontro poderia ser atribuído à presença de danos diretos causados pelo parasito, e quando associado ao tratamento é potencializada pela toxicidade da droga ${ }^{35,36}$. Além disso, infecções parasitárias crônicas estão frequentemente associadas à formação de imunocomplexos que podem se depositar nos glomérulos renais e tubulointersticiais, produzindo glomerulonefrite e nefrite intersticial com o comprometimento da função renal ${ }^{37,38}$.

O edema, um sinal clínico na $L V$, embora sem associação estatística neste estudo (teste exato de Fisher p $>0,05$ ), poderia estar relacionado com o envolvimento renal e a diminuição de proteínas, assim como já observado por Salgado-Filho, Ferreira e Costa ${ }^{39}$.

Outro fator que pode influenciar o curso da doença é a comorbidade, uma condição que pode ocorrer frequentemente em pacientes com $\mathrm{LV}^{25}$.

A desnutrição esteve presente em 32,7\% dos casos, e tem sido considerada um dos principais fatores de risco para o desenvolvimento da forma sintomática da doenç ${ }^{22,40}$. Diversos autores mencionam que a deficiência nutricional afeta particularmente a função fagocítica, produção de anticorpos, citocinas, afinidade do anticorpo para com o antígeno e o sistema complemento o que aumentaria risco de morte ${ }^{41-43}$. Contudo, ainda não está bem esclarecido se a desnutrição é um fator predisponente por fazer parte do estado geral do paciente ou consequência da doença pela ação do parasito que atua como agente coadjuvante, exacerbando o quadro subnutricional pré-existente ${ }^{23,25}$.

O álcool é a droga psicoativa mais utilizada pela humanidade e, neste estudo, 21,8\% dos pacientes relataram ser etilistas crônicos. Assim, o comprometimento hepático, provocado por anos de ingestão alcoólica, pode ter sido agravado pelo intenso parasitismo hepático e a instituição da terapia medicamentosa ${ }^{28,29}$. Além disso, o lento e progressivo comprometimento do organismo pelo alcoolismo também pode ter ocasionado uma diminuição da resposta imune perante este parasito assim como em outras doenças parasitárias ${ }^{44,45}$.

A LV nos últimos anos assumiu caráter de doença oportunista em pessoas infectadas com o HIV e a concomitância de ambas as infecções pode reduzir a sobrevida dos pacientes ${ }^{46,47}$.

Sabe-se que a presença de LV no indivíduo infectado pelo vírus HIV acelera a progressão desta infecção ao promover a replicação viral, agravando ainda mais o estado de imunossupressão ${ }^{46}$. Por outro lado, é observado in vitro que o HIV induz a replicação de Leishmania pela diminuição de células $T$ capazes de reconhecer os antígenos da mesma. Cruz e cols ${ }^{48}$ relatam ainda que, além da Leishmania, o HIV pode invadir e se replicar em macrófagos, mesmo sendo as células TCD $4^{+}$as preferenciais. Deste modo, espera-se uma atuação sinérgica destes patógenos na potencialização dessas infecções em pacientes co-infectados ${ }^{49}$, e este fato pode justificar o óbito de cinco pacientes no presente estudo.

Nesta casuística, chamou a atenção, a ocorrência de tuberculose, citomegalovírus e HTLV apenas em indivíduos co-infectados LV/ HIV, demonstrando que estas patologias oportunistas estavam associadas à presença do vírus HIV e podem ter condicionado a morte imediata e direta do doente ${ }^{46}$.

Em decorrência da ausência de melhora clínica e de reações adversas, que incluíram insuficiência renal, insuficiência hepática, alterações cardíacas e de consciência, pancreatite e hepatite medicamentosa, houve a substituição do antimoniato por anfotericina $\mathrm{B}$, corroborando a afirmação de Santos e cols ${ }^{50}$ de que pacientes com comorbidades ou infecções bacterianas apresentam maior risco de resposta insatisfatória ao tratamento.

As causas que contribuíram para o óbito neste estudo são semelhantes às descritas por outros autores brasileiros ${ }^{20,40}$ demonstrando que apesar do conhecimento prévio das principais causas imediatas de óbito, estas persistem ao longo dos anos.

Os resultados ressaltam a necessidade de profissionais capacitados para o reconhecimento precoce da doença, bem como o monitoramento clínico e laboratorial dos pacientes durante o tratamento da LV a fim de identificar precocemente possíveis complicações.

Sugere-se ainda a necessidade de maior agilidade no diagnóstico laboratorial ao primeiro atendimento clínico e o desenvolvimento de novas drogas com baixa toxicidade, a fim de reduzir a mortalidade por este agravo.

\section{AGRADECIMENTOS}

Os autores agradecem à direção dos Hospitais pela liberação dos prontuários. À Secretaria de Vigilância Epidemiológica do município de Campo Grande-MS pela confirmação dos casos de óbitos. Ao Professor Doutor Albert Schiaveto de Souza pela realização dos testes estatísticos.

\section{CONFLITO DE INTERESSE}

Os autores declaram não haver nenhum tipo de conflito de interesse no desenvolvimento do estudo.

\section{REFERÊNCIAS}

1. Maia-Elkhoury AN, Alves WA, Sousa-Gomes ML, Sena JM, Luna EA. Visceral leishmaniasis in Brazil: trends and challenges. Cad Saude Publica 2008; 24:29412947.

2. Schriefer A, Barral A, Carvalho EM, Barral-Netto M. Serum soluble markers in the evaluation of treatment in human visceral leishmaniasis. Clin Exp Immunol 1995; 102:535-540.

3. Dantas-Torres F, Brandão-Fillho SP. Visceral leishmaniasis in Brazil: revisiting paradigms of epidemiology and control. Rev Inst Med Trop Sao Paulo 2006; 48:151-156.

4. Desjeux P.Leishmaniasis: current situation and new perspectives. Comp Immunol Microbiol Infect Dis 2004; 27:305-318.

5. Pastorino AC, Jacob CMA, Oselka GW, Carneiro-Sampaio MMS. Leishmaniose visceral: aspectos clínicos e laboratoriais. J Pediatr 2002; 78:120-127. 
6. Pedrosa CMS, Rocha EMM. Aspectos clínicos e epidemiológicos da leishmaniose visceral em menores de 15 anos procedentes de Alagoas, Brasil. Rev Soc Bras Med Trop 2004; 37:300-304.

7. Kafetzis DA. An overview of paediatric leishmaniasis. J Postgrad Med 2003; 49:31-38.

8. Werneck GL, Batista MS, Gomes JR, Costa DL, Costa CH. Prognostic factors for death from visceral leishmaniasis in Teresina, Brazil. Infection 2003; 31:174-177.

9. Governo do Estado do Mato Grosso do Sul. Casos Absolutos de Leishmaniose Visceral Americana, Mato Grosso do Sul, 1999-2009 [Internet]. Mato Grosso do Sul (MS): Secretaria de Estado de Saúde. Serviço de Vigilância Epidemiológica. Relatório de Notificações de LV. SINAN; 23 dez 2009 [30 jan 2010]. Disponível em: <http://www.sgi.ms.gov.br/pantaneiro/sites/saude/index. php?templat=vis\&site $=116 \&$ id_comp $=634 \& i d \_r e g=5214 \&$ voltar $=$ lista\&site reg=116\&id_comp_orig=634>.

10. Oliveira ALL, Paniago AMM, Dorval MEC, Leal CR, Sanches M, Cunha RV, et al. Foco emergente de leishmaniose visceral em Mato Grosso do Sul. Rev Soc Bras Med Trop 2006; 39:446-450.

11. Raw I, Cruz O, Schmidt BJ, Diament AJ, Fava-Netto C. Valores de laboratório: referências normais e patológicas sua importância clínica e terapêutica. Rio de Janeiro: Atheneu; 1989.

12. Deane LM. Leishmaniose Visceral no Brasil: estudos sobre reservatórios e transmissores realizados no Estado do Ceará. Rio de Janeiro: Serviço Nacional de Educação Sanitária; 1956.

13. Luz KG, Cabral ALLV, Fiúza VOP, Rabello AA. Urbanização das leishmanioses e a baixa resolutividade diagnóstica em municípios da região metropolitana de Belo Horizonte. Rev Soc Bras Med Trop 2001; 34:249-254.

14. Monteiro EM, Silva JCF, Costa RT, Costa DC, Barata RA, Paula EV, et al. Leishmaniose visceral: estudo de flebotomíneos e infecção canina em Montes Claros, Minas Gerais. Rev Soc Bras Med Trop 2005; 38:147-152.

15. Silva EA, Andreotti R, Honer MR. Comportamento de Lutzomyia longipalpis, vetor principal da leishmaniose visceral americana, em Campo Grande, Estado do Mato Grosso do Sul. Rev Soc Bras Med Trop 2007; 40:420-425.

16. Souza RG, Santos JF, Rodrigues HG, Aversi-Ferreira TA. Casos de leishmaniose visceral registrados no município de Montes Claros, Estado de Minas Gerais. Acta Sci Health Sci 2008; 30:155-159.

17. Oliveira ALL, Paniago AMM, Sanches MA, Dorval ME, Oshiro ET, Leal CR, et al. Asymptomatic infection in family contacts of patients with human visceral leishmaniasis in Três Lagoas, Mato Grosso do Sul State, Brazil. Cad Saúde Pública $2008 ; 24: 2827-2833$

18. Costa CHN, Pereira HF, Araújo MV. Epidemia de leishmaniose visceral no Estado do Piauí, Brasil, 1980-1986. Rev Saude Publica 1990; 24:361-372.

19. Collin S, Davidson R, Ritmeijer K, Keus K, Melaku Y, Kipngetich S, et al. Conflict and kala-azar: determinants of adverse outcomes of kala-azar among patients in southern Sudan. Clin Infect Dis 2004; 38:612-619.

20. Queiroz MJA, Alves JGB, Correia JB. Leishmaniose visceral: características clínicoepidemiológicas em crianças de área endêmica. J Pediatr 2004; 80:141-146.

21. Collin SM, Coleman PG, Ritmeijer K, Davidson RN. Unseen Kala-azar deaths in south Sudan (1999-2002). Trop Med Int Health 2006; 11:509-512.

22. Badaró R, Jones TC, Carvalho EM, Sampaio D, Reed SG, Barral A, et al. New perspectives on a subclinical form of visceral leishmaniasis. J Infect Dis 1986; 154:1003-1011.

23. Seaman J, Mercer AJ, Sondorp HE, Herwaldt BL. Epidemic visceral leishmaniasis in southern Sudan: treatment of severely debilitated patients under wartime conditions and with limited resources. Ann Intern Med 1996; 124:664-672.

24. Cascio A, Colomba C, Antinori S, Orobello M, Paterson D, Titone L. Pediatric visceral leishmaniasis in Western Sicily, Italy: a retrospective analysis of 111 cases. Eur J Clin Microbiol Infect Dis 2002; 21:277-282.

25. Maltezou HC, Siafas C, Mavrikou M, Spyridis P, Stavrinadis C, Karpathios TH, et al. Visceral Leishmaniasis during childhood in Southern Greece. Clin Infect Dis 2000; 31:1139-1143.

26. Barati M, Sharifi I, Daie-Parizi M, Fasihi-Harandi M. Bacterial infections in children with visceral leishmaniasis: observations made in Kerman province, southern Iran, between 1997 and 2007. Ann Trop Med Parasitol 2008; 102:635-641.

27. Kadivar MR, Kajbaf TZ, Karimi A, Alborzi A. Childhood visceral leishmaniasis complicated by bacterial infections. East Mediterr Health J 2000; 6:879-883.
28. Jerônimo SMB, Oliveira RM, Mackay S, Costa RM, Sweet J, Nascimento ET, et al. An urban outbreak of visceral leishmaniasis in Natal, Brazil. Trans R Soc Trop Med Hyg 1994; 88:386-388.

29. Kopke LFF, Café MEM, Neves LB, Scherrer MAR, Pinto-Machado J, Souza MSLA, et al. Morte após uso de antimonial pentavalente em leishmaniose tegumentar americana. An Bras Dermatol 1993; 68:259-261.

30. Ikeda FA, Laurenti MD, Cobertt CE, Feitosa MM, Machado GF, Perri SHV Histological and immunohistochemical study of the central nervous system of dogs naturally infected by Leishmania (Leishmania) chagasi. BrazJ Vet Res Anim Sci 2007; 44:5-11.

31. Meleney HE. The histopathology of kala-azar in the hamster, monkey, and man. Am J Path 1924; 1:147-174.

32. Prasad LSN, Sen S. Migration of Leishmania donovani amastigotes in cerebrospinal fluid. Am J Trop Med Hyg 1996; 55: 652-654.

33. Gasser RAJr, Magill AJ, Oster CN, Franke ED, Grögl M, Berman JD. Pancreatitis induced by pentavalent antimonial agents during treatment of leishmaniasis. Clin Infect Dis 1994; 18:83-90.

34. Santos J, Rivero A, Márquez M. Acute pancreatitis with a fatal evolution due to antimonials in patients with visceral leishmaniasis and HIV infection. An Med Interna 2000; 17:562-563.

35. Caravaca F, Munõz A, Pizarro JL, Santamaría JS, Fernadez-Alonso J. Acute renal failure in visceral leishmaniasis. Am J Nephrol 1991; 11:350-352.

36. Daher EF, Evangelista LF, Silva Júnior GB, Lima RSA, Aragão EB, Arruda GAJC, et al. Clinical presentation and renal evaluation of human visceral leishmaniasis (Kala-azar): A retrospective study of 57 patients in Brazil. Braz J Infect Dis 2008; 12:329-332.

37. Dutra M, Martinelli R, De Carvalho EM, Rodrigues LE, Brito E, Rocha H. Renal involvement in visceral leishmaniasis. Am J Kidney Dis 1985; 6:22-27.

38. Prasad LSN, Sen S, Ganguly SK. Renal involvement in Kala-azar. Indian J Med Res 1991; 95:43-46.

39. Salgado-Filho N, Ferreira TMAF, Costa JML. Envolvimento da função renal em pacientes com leishmaniose visceral (calazar). Rev Soc Bras Med Trop 2003; 36:217-221.

40. Rey LC, Martins CV, Ribeiro HB, Lima AAM. Leishmaniose visceral americana (calazar) em crianças hospitalizadas de área endêmica. J Pediatr 2005; 81:73 78.

41. Gershwin ME, Lentz DR, Beach RS, Hurley LS. Nutritional factors and autoimmunity. IV. Dietary vitamin-A deprivation induces a selective increase in IgM autoantibodies and hypergammaglobulinemia in New-Zealand black mice. J Immunol 1984; 133:222-226.

42. Chandra RK. Nutrition and immunoregulation - significance for host resistance totumors and infectious diseases in humans and rodents. J Nutr 1992; 122:754 757.

43. Fock RA, Vinolo MA, Rocha VMS, Rocha LCS, Borelli P. Protein-energy malnutrition decreases the expression of TLR-4/MD-2 and CD14 receptors in peritoneal macrophages and reduces the synthesis of TNF-á in response to lipopolysaccharide (LPS) in mice. Cytokine 2007; 40:105-114.

44. Maia TMC, Vasconcelos PRL, Fauth S, Neto RM. Hiperinfestação por Strongyloides stercoralis. RBPS 2006; 19:118-121.

45. Oliveira LCM, Ribeiro CT, Mendes DM, Oliveira TC, Costa-Cruz JM. Frequency of Strongyloides stercoralis infection in alcoholics. Mem Inst Oswaldo Cruz 2002; 97:119-121.

46. Chaisson RE, Gallant JE, Keruly JC, Moore RD. Impact of opportunistic disease on survival in patients with HIV infection. AIDS 1998; 12: 29-33.

47. Choi CM, Lerner EA. Leishmaniasis as an emerging infection.J Investig Dermatol Symp Proc 2001; 6:175-182.

48. Cruz I, Nieto J, Moreno J, Cañavate C, Desjeux P, Alvar J. Leishmania/HIV coinfections in the second decade. Indian J Med Res 2006; 123:357-388.

49. Wolday D, Akuffo H, Fessahaye G, Valantine A, Britton S. Live and killed human immunodeficiency virus type-1 increases the intracellular growth of Leishmania donovani in monocyte-derived cells. Scand J Infect Dis 1998; 30:29-34.

50. Santos MA, Marques RC, Farias CA, Vasconcelos DM, Stewart JM, Costa CHN Predictors of an unsatisfactory response to pentavalent antimony in the treatment of American visceral leishmaniasis. Rev Soc Bras Med Trop 2002; 35:629-633. 